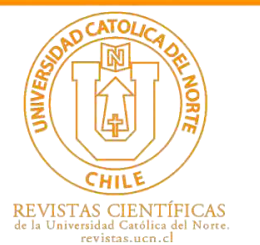

\title{
The endomorphisms algebra of translations group and associative unitary ring of trace-preserving endomorphisms in affine plane*
}

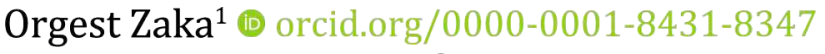 \\ Mohanad A. Mohammed ${ }^{2}$ (D) orcid.org/0000-0002-7507-1212
}

\begin{abstract}
${ }^{1}$ Agricultural University of Tirana, Dept. of Mathematics-Informatics, Tirana, Albania. - ozaka@ubt.edu.al

${ }^{2}$ Open Educational College, Dept. of Mathematics, Al Qadisiya Centre, Ministry of Education, Amarah, Iraq. mohanadalim@gmail.com
\end{abstract}

Received: February 2020 | Accepted: May 2020

\begin{abstract}
:
A description of Endomorphisms of the translation group is introduced in an affine plane, will define the addition and composition of the set of endomorphisms and specify the neutral elements associated with these two actions and present the Endomorphism algebra thereof will distinguish the Trace-preserving endomorphism algebra in affine plane, and prove that the set of Trace-preserving endomorphism associated with the 'addition' action forms a commutative group. We also try to prove that the set of trace-preserving endomorphism, together with the two actions, in it, 'addition' and 'composition' forms an associative and unitary ring. act value of edge irregularity strength of different families of comb graph.
\end{abstract}

Keywords:Affine plane; Endomorphisms; Trace-preserving endomorphisms; Translation group; Aditive group; Associative ring

MSC (2020): 51-XX, 51Axx, 51A25, 51A40, 08Axx, 16-XX, 16Sxx, $16 S 50$.

\section{Cite this article as (IEEE citation style):}

O. Zaka and M. A. Mohammed, "The endomorphisms algebra of translations group and associative unitary ring of trace-preserving endomorphisms in affine plane", Proyecciones (Antofagasta, On line), vol. 39, no. 4, pp. 821-834, Aug. 2020, doi: 10.22199/issn.0717-6279-2020-04-0051.

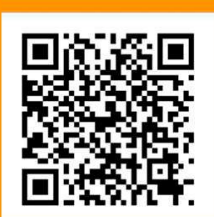

Article copyright: (C) 2020 Orgest Zaka and Mohanad. A. Mohammed. This is an open access article distributed under the terms of the Creative Commons License, which permits unrestricted use and distribution provided the original author and source are credited.

*Dedicated to David Hilbert and Emil Artin

(cc) BY 


\section{Introduction}

The foundations for the study of the connections between axiomatic geometry and algebraic structures were set forth by D. Hilbert [7], recently elaborated and extended in terms of the algebra of affine planes in, for example, [9], [4, IX.3, p.574]. Also great contributions in this direction have been made by, E.Artin in [1], H. S. M. Coxeter, in [4], Marcel Berger in [2,3], Robin Hartshrone in [6], etc. Even earlier, in my works [5,13,14,15,16,17,18, 19,20 ] I have brought up quite a few interesting facts about the association of algebraic structures with affine planes and with 'Desargues affine planes', and vice versa.

In this paper, we will present a description of Endomorphisms of the translation group in affine plane. We will define the addition and composition of the set of endomorphisms of the translation group in the affine plane. We will specify the neutral elements associated with these two actions, which we will call, 'zero endomorphism' and 'unitary endomorphism', and present the Endomorphism algebra of translations in affine plane. We will distinguish, as a substructure of Endomorphisms algebra, the Tracepreserving endomorphism algebra in affine planes, and prove that the set of Trace-preserving endomorphism associated with the 'addition' action forms a commutative group, also prove that the set of trace-preserving endomorphism, together with the two actions, in it, 'addition' and 'composition' forms an associative and unitary ring.

In paper [20], we have done a detailed, careful description, of translations and dilations in affine planes. We have proven that the set of dilations regarding the composition action is a group, and set of translations is a commutative group. We have proved that translation group is a normal subgroup of the group of dilations. We have described and specified what we would call a translation or dilation trace, we have also defined the direction of an translation as an equivalence class of parallelism in affine planes. This will help us in this paper, as trace-preserving endomorphisms to retain those endomorphisms that operate on these equivalence classes according to parallelism.

\section{Preliminaries}

Let $\mathcal{P}$ be a nonempty set, which is called set of points, $\mathcal{L}$ a nonempty subset of $\mathcal{P}$, which is called set of lines, and an incidence relation $\mathcal{I} \subseteq \mathcal{P} \times \mathcal{L}$. For a point $P \in \mathcal{P}$ and an line $\ell \in \mathcal{L}$, the fact $(P, \ell) \in \mathcal{I}$, (equivalent to $P \mathcal{I} \ell$ ) 
we mark $P \in \ell$ and read point $P$ is incident with a line $\ell$ or a line passes through points $P$ (contains point $P$ ).

Definition 1. [1], [8], [16] Affine plane is called the incidence structure $\mathcal{A}=$ $(\mathcal{P}, \mathcal{L}, \mathcal{I})$ that satisfies the following axioms:

A.1 For every two different points $P$ and $Q \in \mathcal{P}$, there exists exactly one line $\ell \in \mathcal{L}$ incident with that points.

A.2 For a point $P \in \mathcal{P}$, and an line $\ell \in \mathcal{L}$ such that $(P, \ell) \notin \mathcal{I}$, there exists one and only one line $r \in \mathcal{L}$, incident with the point $P$ and such that $\ell \cap r=\emptyset$.

A.2 In $\mathcal{A}$ there are three non-incident points with a line.

Whereas a line of the affine plane we consider as sets of points of affine plane with her incidents. Axiom A.1 implicates that tow different lines of $\mathcal{L}$ many have a common point, in other words tow different lines of $\mathcal{L}$ either have no common point or have only one common point.

Definition 2. Two lines $\ell, m \in \mathcal{L}$ that are matching or do not have common point are called parallel and in this case is written $\ell \| m$, and when they have only one common point we say that they are expected.

Definition 3. Let it be $\mathcal{A}=(\mathcal{P}, \mathcal{L}, \mathcal{I})$ an affine plane and $\mathcal{S}=\{\psi: \mathcal{P} \rightarrow$ $\mathcal{P} \mid$ where $\psi$-is bijection $\}$ set of bijections to set points $\mathcal{P}$ on yourself. Collineation of affine plane $\mathcal{A}$, called a bijection $\psi \in \mathcal{S}$, such that

$$
\forall \ell \in \mathcal{L}, \psi(\ell) \in \mathcal{L},
$$

Otherwise, a collineation of the affine plane $\mathcal{A}$ is a bijection of set $\mathcal{P}$ on yourself, that preserves lines. It is known that the set of bijections to a set over itself is a group on associated with the binary action ' $\circ$ ' of composition in it, which is known as total group or symmetric groups.

Definition 4. [20] An point $P$ of the affine plane $\mathcal{A}$ called fixed point his associated with a collineation $\delta$, if coincides with the image itself $\delta(P)$, briefly when, $P=\delta(P)$.

Definition 5. $[1,20]$ A Dilation of an affine plane $\mathcal{A}=(\mathcal{P}, \mathcal{L}, \mathcal{I})$ called a its collineation $\delta$ such that

$$
\forall P \neq Q \in \mathcal{P}, \delta(P Q) \| P Q
$$


Let it be $\operatorname{Dil}_{\mathcal{A}}=\left\{\delta \in \mathbf{C o l}_{\mathcal{A}} \mid \delta\right.$ - is a dilation of $\left.\mathcal{A}\right\}$ the dilation set of affine plane $\mathcal{A}=(\mathcal{P}, \mathcal{L}, \mathcal{I})$.

Theorem 1. $[20,13]$ The dilation set $\operatorname{Dil}_{\mathcal{A}}$ of affine plane $\mathcal{A}$ forms a group with respect to composition $\circ$.

Proof. See, Theorem 2.4, in [20].

Definition 6. Let it be $\delta$ an dilation of affine plane $\mathcal{A}=(\mathcal{P}, \mathcal{L}, \mathcal{I})$, and $P$ a point in it. Lines that passes by points $P$ and $\delta(P)$, called trace of points $P$ regarding dilation $\delta$.

Every point of a traces of a not-fixed point, to an affine plane associated with its dilation has its own image associated with that dilation in the same traces (see [20]). We also know the result: If an affine plane $\mathcal{A}=(\mathcal{P}, \mathcal{L}, \mathcal{I})$, has two fixed points about an dilation then he dilation is identical dilation $i d_{\mathcal{P}}$ of his (Theorem 2.12 in $[20]$ ).

In an affine plane related to dilation $\delta \neq i d_{\mathcal{P}}$ all traces $P \delta(P)$ for all $P \in \mathcal{P}$, or cross the by a single point, or are parallel between themselves (see [12], [20]).

Definition 7. $[1,20]$ The Translation of an affine plane $\mathcal{A}=(\mathcal{P}, \mathcal{L}, \mathcal{I})$, called the his identical dilation $i d_{\mathcal{P}}$ and every other of it's dilation, about which that the affine plane has not fixed points.

If $\sigma$ is an translation different from identical translation $i d_{\mathcal{P}}$, then, all traces related to $\sigma$ form the a set of parallel lines.

Definition 8. For one translation $\sigma \neq i d_{\mathcal{P}}$, the parallel equivalence classes of the cleavage $\pi=\mathcal{L} / \|$, which contained tracks by $\sigma$ of points of the plane $\mathcal{A}=(\mathcal{P}, \mathcal{L}, \mathcal{I})$ called the direction of his translation $\sigma$ and marked with $\pi \cdot 0 \cdot \sigma$.

So, for $\sigma \neq i d_{\mathcal{P}}$, the direction $\pi_{\sigma}$ represented by single the trace (which is otherwise called, representative of direction) by $\sigma$ for every point $P \in \mathcal{P}$, for translation $i d_{\mathcal{P}}$ has undefined direction.

Let it be $\alpha: \operatorname{Tr}_{\mathcal{A}} \longrightarrow \operatorname{Tr}_{\mathcal{A}}$, an whatever map of $\operatorname{Tr}_{\mathcal{A}}$, on yourself. For every translation $\sigma$, its image $\alpha(\sigma)$ is again an translation, that can be $\alpha(\sigma)=i d_{\mathcal{P}}$ or $\alpha(\sigma) \neq i d_{\mathcal{P}}$. So there is a certain direction or indefinite. The first equation, in the case where $\sigma=i d_{\mathcal{P}}$, takes the view $\alpha\left(i d_{\mathcal{P}}\right)=i d_{\mathcal{P}}$, 
and the second $\alpha(\sigma) \neq i d_{\mathcal{P}}$, that it is not possible to $\alpha$ is map. To avoid this, yet accept that for every map $\alpha: \operatorname{Tr}_{\mathcal{A}} \longrightarrow \operatorname{Tr}_{\mathcal{A}}$, is true this,

$$
\alpha\left(i d_{\mathcal{P}}\right)=i d_{\mathcal{P}}
$$

Proposition 1. [20] If translations $\sigma_{1}$ and $\sigma_{2}$ have the same direction with translation $\sigma$ to an affine plane $\mathcal{A}=(\mathcal{P}, \mathcal{L}, \mathcal{I})$, then and composition $\sigma_{2} \circ \sigma_{1}$ has the same the direction, otherwise

$$
\forall \sigma_{1}, \sigma_{2}, \sigma \in \operatorname{Tr}_{\mathcal{A}}, \pi_{\sigma_{1}}=\pi_{\sigma_{2}}=\pi_{\sigma} \Longrightarrow \pi_{\sigma_{2} \circ \sigma_{1}}=\pi_{\sigma}
$$

Theorem 2. [20] Set $\operatorname{Tr}_{\mathcal{A}}$ of translations to an affine plane $\mathcal{A}$ form a group about the composition $\circ$, which is a sub-group of the group $\left(\mathbf{D i l}_{\mathcal{A}}, \circ\right)$ to dilations of affine plane $\mathcal{A}$.

Theorem 3. [20] Group $\left(\operatorname{Tr}_{\mathcal{A}}, \circ\right)$ of translations to the affine plane $\mathcal{A}$ is normal sub- group of the group of dilations $\left(\mathbf{D i l}_{\mathcal{A}}, \circ\right)$ of him plane.

$$
\forall \delta \in D i l_{\mathcal{A}}, \forall \sigma \in \operatorname{Tr}_{\mathcal{A}} \Rightarrow \delta^{-1} \circ \sigma \circ \delta \in \operatorname{Tr}_{\mathcal{A}}
$$

Corollary 1. [20] For every dilation $\delta \in \mathbf{D i l}_{\mathcal{A}}$ and for every translations $\sigma \in \operatorname{Tr}_{\mathcal{A}}$ of affine plane $\mathcal{A}=(\mathcal{P}, \mathcal{L}, \mathcal{I})$, translations $\sigma$ and $\delta^{-1} \circ \sigma \circ \delta$ of his have the same direction.

Corollary 2. The translations group $\left(\operatorname{Tr}_{\mathcal{A}}, \circ\right)$ of an affine plane $\mathcal{A}$ is (Abelian) commutative Group.

\section{The Endomorphisms Algebra, of the Translation's Group and their trace-preserving Associative Unitary Ring}

Consider the affine plane $\mathcal{A}=(\mathcal{P}, \mathcal{L}, \mathcal{I})$ and set of maps, of commutative $\operatorname{Group}\left(T r_{\mathcal{A}}, \circ\right)$ of affine plane $\mathcal{A}$ in itself (see [20]), so $\left(T r_{\mathcal{A}}\right)^{T r_{\mathcal{A}}}=$ $\left\{\alpha \mid \alpha: \operatorname{Tr}_{\mathcal{A}} \rightarrow \operatorname{Tr}_{\mathcal{A}}\right\}$. Let be $\alpha, \beta$ two different maps, such. Then for every $\sigma \in \operatorname{Tr}_{\mathcal{A}} \Rightarrow \alpha(\sigma) \in \operatorname{Tr}_{\mathcal{A}}, \beta(\sigma) \in \operatorname{Tr}_{\mathcal{A}}$ and $[\alpha \circ \beta](\sigma)=\alpha(\beta(\sigma)) \in \operatorname{Tr}_{\mathcal{A}}$. From the latter it turns out that the action of composition 'o' in the set $\left(T r_{\mathcal{A}}\right)^{T r_{\mathcal{A}}}$, is action induced by the action of composition ' $\mathrm{o}$ ' in the set $(\mathcal{P})^{\mathcal{P}}$, of maps $\mathcal{P} \rightarrow \mathcal{P}$ of the affine plane $\mathcal{A}$. If the associate translations $\sigma$, the unique translation $\alpha(\sigma) \circ \beta(\sigma)$, obtained a new map $\operatorname{Tr}_{\mathcal{A}} \rightarrow \operatorname{Tr}_{\mathcal{A}}$, which we call addition of $\alpha$ with $\beta$, and mark with $\alpha+\beta$. 
Definition 9. For every two maps $\alpha, \beta \in\left(\operatorname{Tr}_{\mathcal{A}}\right)^{T r_{\mathcal{A}}}$, the addition of them, that is marked $\alpha+\beta$, is called the map ' $\alpha+\beta: \operatorname{Tr}_{\mathcal{A}} \rightarrow \operatorname{Tr}_{\mathcal{A}}$ ', defined by,

$$
(\alpha+\beta)(\sigma)=(\alpha)(\sigma) \circ(\beta)(\sigma), \forall \sigma \in \operatorname{Tr}_{\mathcal{A}} .
$$

Accompanying any two maps $\alpha, \beta$ their sum $\alpha+\beta$, we obtain a new binary action in $\operatorname{Tr}_{\mathcal{A}}$, that we call the addition of maps, of translations in the affine plane $\mathcal{A}$.

Thus obtained, algebra with two binary operations $\left(\left(\operatorname{Tr}_{\mathcal{A}}\right)^{T r_{\mathcal{A}}},+, \mathrm{o}\right)$, where the sum of the two elements, whatsoever, its $\alpha, \beta$ in $\left(\operatorname{Tr}_{\mathcal{A}}\right)^{T r_{\mathcal{A}}}$, is given by Definition 9 , and their composition is given by,

$$
[\alpha \circ \beta](\sigma)=\alpha(\beta(\sigma)), \forall \sigma \in \operatorname{Tr}_{\mathcal{A}}
$$

Definition 10. The Algebra $\left(\left(T r_{\mathcal{A}}\right)^{T r_{\mathcal{A}}},+, \circ\right)$, is called the algebra of, maps of $\operatorname{Tr}_{\mathcal{A}}$ on himself.

A map $\alpha: \operatorname{Tr}_{\mathcal{A}} \longrightarrow \operatorname{Tr}_{\mathcal{A}}$, is an endomorphism of the group $\left(\operatorname{Tr}_{\mathcal{A}}, \circ\right)$, on himself (see [16], [13]), namely such that,

$$
\forall \sigma_{1}, \sigma_{2} \in \operatorname{Tr}_{\mathcal{A}}, \alpha\left(\sigma_{1} \circ \sigma_{2}\right)=\alpha\left(\sigma_{1}\right) \circ \alpha\left(\sigma_{2}\right) .
$$

Lemma 1. The addition of, each two endomorphisms of $\operatorname{Tr}_{\mathcal{A}}$ on himself, is a endomorphisms of $\operatorname{Tr}_{\mathcal{A}}$ on himself.

Proof. Let them be $\alpha, \beta$ two endomorphisms of $\operatorname{Tr}_{\mathcal{A}}$ on himself. According to $6, \forall \sigma_{1}, \sigma_{2} \in \operatorname{Tr}_{\mathcal{A}}$ have,

$$
\alpha\left(\sigma_{1} \circ \sigma_{2}\right)=\alpha\left(\sigma_{1}\right) \circ \alpha\left(\sigma_{2}\right) \text { and } \beta\left(\sigma_{1} \circ \sigma_{2}\right)=\beta\left(\sigma_{1}\right) \circ \beta\left(\sigma_{2}\right) .
$$

Then, we have:

$$
\begin{aligned}
{[\alpha+\beta]\left(\sigma_{1} \circ \sigma_{2}\right) } & =\alpha\left(\sigma_{1} \circ \sigma_{2}\right) \circ \beta\left(\sigma_{1} \circ \sigma_{2}\right)(b y, 4) \\
& =\left[\alpha\left(\sigma_{1}\right) \circ \alpha\left(\sigma_{2}\right)\right] \circ\left[\beta\left(\sigma_{1}\right) \circ \beta\left(\sigma_{2}\right)\right](b y, 6) \\
& \left.=\left[\alpha\left(\sigma_{1}\right) \circ \beta\left(\sigma_{1}\right)\right] \circ\left[\alpha\left(\sigma_{2}\right)\right] \circ \beta\left(\sigma_{2}\right)\right](b y, 4) \\
& =[\alpha+\beta]\left(\sigma_{1}\right) \circ[\alpha+\beta]\left(\sigma_{2}\right)(b y, 4) .
\end{aligned}
$$

Thus,

$$
\begin{gathered}
\forall \sigma_{1}, \sigma_{2} \in \operatorname{Tr}_{\mathcal{A}}, \\
{[\alpha+\beta]\left(\sigma_{1} \circ \sigma_{2}\right)=[\alpha+\beta]\left(\sigma_{1}\right) \circ[\alpha+\beta]\left(\sigma_{2}\right) .}
\end{gathered}
$$

Lemma 2. The composition of any two endomorphisms of $\operatorname{Tr}_{\mathcal{A}}$ on himself, is an endomorphisms of $\operatorname{Tr}_{\mathcal{A}}$ on himself. 
Proof. In the conditions when $\alpha$ and $\beta$ are endomorphisms, by eq. 5 we have too

$$
\begin{aligned}
{[\alpha \circ \beta]\left(\sigma_{1} \circ \sigma_{2}\right) } & =\alpha\left[\beta\left(\sigma_{1} \circ \sigma_{2}\right)\right](b y, 5) \\
& =\alpha\left[\beta\left(\sigma_{1}\right) \circ \beta\left(\sigma_{2}\right)\right](b y, 6) \\
& =\alpha\left[\beta\left(\sigma_{1}\right)\right] \circ\left[\beta\left(\sigma_{2}\right)\right](b y, 6) \\
& =[\alpha \circ \beta]\left(\sigma_{1}\right) \circ[\alpha \circ \beta]\left(\sigma_{2}\right)(b y, 5)
\end{aligned}
$$

Thus,

$$
\begin{gathered}
\forall \sigma_{1}, \sigma_{2} \in \operatorname{Tr}_{\mathcal{A}}, \\
{[\alpha \circ \beta]\left(\sigma_{1} \circ \sigma_{2}\right)=[\alpha \circ \beta]\left(\sigma_{1}\right) \circ[\alpha \circ \beta]\left(\sigma_{2}\right) .}
\end{gathered}
$$

From Lemmas $1 \& 2$, based on the understanding of a substructure of an algebraic structure (see [10], [11], [12]), we get this too,

Theorem 1. The set of endomorphisms of $\operatorname{Tr}_{\mathcal{A}}$ on himself, regarding actions 'addition +' and 'composition o' in ti, is a substructure of algebra $\left(\left(T r_{\mathcal{A}}\right)^{T r_{\mathcal{A}}},+, \circ\right)$ of maps, of $\operatorname{Tr}_{\mathcal{A}}$ on oneself.

We call it, the 'endomorphisms-algebra' of the $\operatorname{Tr}_{\mathcal{A}}$ group on ourselves and mark with $E n d_{T r_{\mathcal{A}}}$.

Definition 11. The 'tracer-preserving' endomorphism of the group $\left(\operatorname{Tr}_{\mathcal{A}}, \circ\right)$ above itself, it is called an endomorphism $\alpha \in E n d_{T r_{\mathcal{A}}}$ his, such that

$$
\forall \sigma \in \operatorname{Tr}_{\mathcal{A}}, \pi_{\alpha(\sigma)}=\pi_{\sigma},
$$

otherwise, any trace according to $\alpha(\sigma)$ is a trace according to $\sigma$.

The 'tracer-preserving' endomorphism of the group $\left(T r_{\mathcal{A}}, \circ\right)$ above itself, we will marked with, $E n d_{T r_{\mathcal{A}}}^{T P}$

The map $0_{\operatorname{Tr}_{\mathcal{A}}}: \operatorname{Tr}_{\mathcal{A}} \longrightarrow \operatorname{Tr}_{\mathcal{A}}$, determined by

$$
0_{\operatorname{Tr}_{\mathcal{A}}}(\sigma)=i d_{\mathcal{P}}, \forall \sigma \in \operatorname{Tr}_{\mathcal{A}} .
$$

is an endomorphism of the translation group $\operatorname{Tr}_{\mathcal{A}}$ on himself because,

$$
\begin{gathered}
\forall \sigma_{1}, \sigma_{2} \in \operatorname{Tr}_{\mathcal{A}}, \\
0_{T r_{\mathcal{A}}}\left(\sigma_{1} \circ \sigma_{2}\right)=i d_{\mathcal{P}}=i d_{\mathcal{P}} \circ i d_{\mathcal{P}}=0_{T r_{\mathcal{A}}}\left(\sigma_{1}\right) \circ 0_{T r_{\mathcal{A}}}\left(\sigma_{2}\right) .
\end{gathered}
$$

So,

$$
0_{T r_{\mathcal{A}}} \in E n d_{T r_{\mathcal{A}}}
$$


The endomorphism $0_{T r_{\mathcal{A}}}$ call it, zero endomorphism of $\operatorname{Tr}_{\mathcal{A}}$ on himself.

From eq. 8 also indicate that,

$$
\forall \sigma \in \operatorname{Tr}_{\mathcal{A}}, \pi_{0_{T_{\mathcal{A}}}(\sigma)}=\pi_{\sigma}
$$

so we have that,

$$
0_{T r_{\mathcal{A}}} \in \operatorname{End}_{\operatorname{Tr}_{\mathcal{A}}}^{T P}
$$

thus is i true this,

Proposition 2. The zero endomorphism $0_{\operatorname{Tr}_{\mathcal{A}}}$ of $\operatorname{Tr}_{\mathcal{A}}$ on himself, is a trace-preserving endomorphism of $\operatorname{Tr}_{\mathcal{A}}$ on himself.

The identical map $1_{T r_{\mathcal{A}}}: \operatorname{Tr}_{\mathcal{A}} \longrightarrow \operatorname{Tr}_{\mathcal{A}}$, defined by

$$
1_{\operatorname{Tr}_{\mathcal{A}}}(\sigma)=\sigma, \forall \sigma \in \operatorname{Tr}_{\mathcal{A}}
$$

is also, an endomorphism of the translation group $\operatorname{Tr}_{\mathcal{A}}$ on himself because,

$$
\begin{gathered}
\forall \sigma_{1}, \sigma_{2} \in \operatorname{Tr}_{\mathcal{A}}, \\
1_{T r_{\mathcal{A}}}\left(\sigma_{1} \circ \sigma_{2}\right)=\sigma_{1} \circ \sigma_{2}=1_{\operatorname{Tr}_{\mathcal{A}}}\left(\sigma_{1}\right) \circ 1_{T r_{\mathcal{A}}}\left(\sigma_{2}\right) .
\end{gathered}
$$

So,

$$
1_{T r_{\mathcal{A}}} \in \operatorname{End}_{\operatorname{Tr} r_{\mathcal{A}}}
$$

The endomorphism $1_{T r_{\mathcal{A}}}$ call it, unitary endomorphism of $\operatorname{Tr}_{\mathcal{A}}$ on himself. From 9 we have immediately that

$$
\forall \sigma \in \operatorname{Tr}_{\mathcal{A}}, \pi_{1_{T_{\mathcal{A}}}(\sigma)}=\pi_{\sigma},
$$

so we have that,

$$
1_{T r_{\mathcal{A}}} \in \operatorname{End}_{\operatorname{Tr}_{\mathcal{A}}}^{T P}
$$

thus is i true this,

Proposition 3. The unitary endomorphism ' $1_{T r_{\mathcal{A}}}$ ' of $T r_{\mathcal{A}}$ on himself, is a trace-preserving endomorphism of $\operatorname{Tr}_{\mathcal{A}}$ on himself.

Theorem 2. If $\alpha$ and $\beta$ are two trace-preserving endomorphisms of $\operatorname{Tr}_{\mathcal{A}}$ on himself, then their sum $\alpha+\beta$ is an trace-preserving endomorphism. 
Proof. In terms of Theorem, according to Lemma 1, the addition $\alpha+\beta$ is an endomorphisms of $\operatorname{Tr}_{\mathcal{A}}$ on himself. We note $\sigma_{1}=\alpha(\sigma)$ and $\sigma_{2}=\beta(\sigma)$. Given that $\alpha$ and $\beta$ are two trace-preserving endomorphisms, according to eq. $7, \forall \sigma \in \operatorname{Tr}_{\mathcal{A}}$ have,

$$
\pi_{\alpha(\sigma)}=\pi_{\sigma_{1}}=\pi_{\sigma} \text { and } \pi_{\beta(\sigma)}=\pi_{\sigma_{2}}=\pi_{\sigma}
$$

From here, according to 3 we get

$$
\forall \sigma \in \operatorname{Tr}_{\mathcal{A}}, \pi_{\alpha(\sigma) \circ \beta(\sigma)}=\pi_{\sigma_{1} \circ \sigma_{2}}=\pi_{\sigma}
$$

Then, by eq. 4 and eq. 11, have that

$$
\pi_{(\alpha+\beta)(\sigma)}=\pi_{\alpha(\sigma) \circ \beta(\sigma)}=\pi_{\sigma}
$$

Hence,

$$
\forall \sigma \in \operatorname{Tr}_{\mathcal{A}}, \pi_{(\alpha+\beta)(\sigma)}=\pi_{\sigma}
$$

Theorem 3. If $\alpha$ and $\beta$ are two trace-preserving endomorphisms of $\operatorname{Tr}_{\mathcal{A}}$ on himself, then their composition $\alpha \circ \beta$ is an trace-preserving endomorphism.

Proof. In terms of Theorem, according to Lemma 2, the composition $\alpha \circ \beta$ is an endomorphisms of $\operatorname{Tr}_{\mathcal{A}}$ on himself. We note $\sigma_{1}=\beta(\sigma)$, given that $\alpha$ and $\beta$ are two trace-preserving endomorphisms, according to eq. 7, $\forall \sigma \in \operatorname{Tr}_{\mathcal{A}}$ and for $\sigma_{1}=\beta(\sigma)$, have

$$
\pi_{\alpha\left(\sigma_{1}\right)}=\pi_{\sigma_{1}} \text { and } \pi_{\beta(\sigma)}=\pi_{\sigma}
$$

Then, by eq. 5 and eq. 12 , have that

$$
\pi_{(\alpha \circ \beta)(\sigma)}=\pi_{\alpha(\beta(\sigma))}=\pi_{\alpha\left(\sigma_{1}\right)}=\pi_{\sigma_{1}}=\pi_{\beta(\sigma)}=\pi_{\sigma} .
$$

Hence,

$$
\forall \sigma \in \operatorname{Tr}_{\mathcal{A}}, \pi_{(\alpha \circ \beta)(\sigma)}=\pi_{\sigma}
$$

We note now, with $\varphi: \operatorname{Tr}_{\mathcal{A}} \longrightarrow \operatorname{Tr}_{\mathcal{A}}$, defined by

$$
\forall \sigma \in \operatorname{Tr}_{\mathcal{A}}, \varphi(\sigma)=\sigma^{-1}
$$

is an endomorphism of the commutative group of translations $\operatorname{Tr}_{\mathcal{A}}$ on himself, because $\forall \sigma_{1}, \sigma_{2} \in \operatorname{Tr}_{\mathcal{A}}$, we have 


$$
\begin{aligned}
\varphi\left(\sigma_{1} \circ \sigma_{2}\right) & =\left(\sigma_{1} \circ \sigma_{2}\right)^{-1}(b y, 13) \\
& =\left(\sigma_{2}\right)^{-1} \circ\left(\sigma_{1}\right)^{-1} \\
& =\left(\sigma_{1}\right)^{-1} \circ\left(\sigma_{2}\right)^{-1}(b y, 2) \\
& =\varphi\left(\sigma_{1}\right) \circ \varphi\left(\sigma_{2}\right)
\end{aligned}
$$

Also, we have that,

$$
\forall \sigma \in \operatorname{Tr}_{\mathcal{A}}, \pi_{\sigma}=\pi_{\sigma^{-1}} \Rightarrow \pi_{\varphi(\sigma)}=\pi_{\sigma^{-1}}=\pi_{\sigma}
$$

from this, we have prove this

Proposition 4. The endomorphisms $\varphi: \operatorname{Tr}_{\mathcal{A}} \longrightarrow \operatorname{Tr}_{\mathcal{A}}$, defined by $\varphi(\sigma)=$ $\sigma^{-1}, \forall \sigma \in \operatorname{Tr}_{\mathcal{A}}$, is an trace-preserving endomorphisms of $\operatorname{Tr}_{\mathcal{A}}$ on himself.

Proposition 5. For a trace-preserving endomorphism $\alpha \in E n d_{T r_{\mathcal{A}}}^{T P}$, the map $-\alpha: \operatorname{Tr}_{\mathcal{A}} \longrightarrow \operatorname{Tr}_{\mathcal{A}}$, defined by,

$$
\forall \sigma \in \operatorname{Tr}_{\mathcal{A}},(-\alpha)(\sigma)=(\alpha(\sigma))^{-1} \Rightarrow(-\alpha) \in \operatorname{End}_{\operatorname{Tr}_{\mathcal{A}}^{T P}}^{T P}
$$

well $-\alpha$ is an trace-preserving endomorphism of $\operatorname{Tr}_{\mathcal{A}}$ on himself.

Proof. Note that $\forall \sigma \in \operatorname{Tr}_{\mathcal{A}}$,

$$
\begin{aligned}
(-\alpha)(\sigma) & =(\alpha(\sigma))^{-1} \\
& =\varphi(\alpha(\sigma))(b y, 13) \\
& =(\varphi \circ \alpha)(\sigma)(b y, 5)
\end{aligned}
$$

which indicates that, $-\alpha=\varphi \circ \alpha$ and according to Theorem 6 , he is a trace-preserving endomorphism.

The endomorphism ${ }^{\prime}-\alpha^{\prime}$ we call it the additive inverse endomorphism of endomorphism $\alpha$. Consider now the set

$$
E n d_{T r_{\mathcal{A}}}^{T P}=\left\{\alpha \in E n d_{T r_{\mathcal{A}}} \mid \alpha-\text { is a trace-preserving endomrphism }\right\}
$$

of trace-preserving endomorphisms of $\operatorname{Tr}_{\mathcal{A}}$ in itself. According to Theorem 5 and Theorem $6,\left(E n d_{\mathcal{A}}^{T P},+, \circ\right)$ is a substructure of the algebra $\left(E n d_{\mathcal{A}},+, \circ\right)$, of endomorphisms related to addition and composition actions, therefore it is itself an algebra.

Theorem 4. The Grupoid $\left(\operatorname{End}_{T r_{\mathcal{A}}}^{T P},+\right)$, is commutative (Abelian) Group. 
Proof. 1. $\forall \alpha, \beta, \gamma \in \operatorname{End}_{T r_{\mathcal{A}}}^{T P}$, have

$$
\begin{aligned}
\forall \sigma \in \operatorname{Tr}_{\mathcal{A}},[(\alpha+\beta)+\gamma](\sigma) & =(\alpha+\beta)(\sigma) \circ(\gamma)(\sigma)(b y, 4) \\
& =[\alpha(\sigma) \circ(\beta)(\sigma)] \circ(\gamma)(\sigma)(b y, 4) \\
& =\alpha(\sigma) \circ[(\beta)(\sigma) \circ(\gamma)(\sigma)](b y, 2) \\
& =\alpha(\sigma) \circ[(\beta+\gamma)(\sigma)](b y, 4) \\
& =[\alpha+(\beta+\gamma)](\sigma)(b y, 4)
\end{aligned}
$$

So,

$$
\forall \alpha, \beta, \gamma \in E_{n d_{T r_{\mathcal{A}}}^{T P}}^{T P}(\alpha+\beta)+\gamma=\alpha+(\beta+\gamma) .
$$

$1 \alpha \in E n d_{T r_{\mathcal{A}}}^{T P}$, have

$$
\begin{aligned}
\forall \sigma \in \operatorname{Tr}_{\mathcal{A}},\left[\alpha+0_{T r_{\mathcal{A}}}\right](\sigma) & =\alpha(\sigma) \circ 0_{T r_{\mathcal{A}}}(\sigma)(b y, 4) \\
& =\alpha(\sigma) \circ i d_{\mathcal{P}}(b y, 8) \\
& =\alpha(\sigma)
\end{aligned}
$$

So exists, the zero element in $E n d_{T r_{\mathcal{A}}}^{T P}$, which is zero endomorphism $0_{T r_{\mathcal{A}}}$ for which, we have proven that it is a trace-preserving endomorphism, and we have that,

$$
\forall \alpha \in E n d_{T r_{\mathcal{A}}}^{T P}, \exists 0_{T r_{\mathcal{A}}} \in E n d_{T r_{\mathcal{A}}}^{T P}, \alpha+0_{T r_{\mathcal{A}}}=0_{\operatorname{Tr}_{\mathcal{A}}}+\alpha=\alpha .
$$

3. $\forall \alpha \in E n d_{T r_{\mathcal{A}}}^{T P}$, have

$$
\begin{aligned}
\forall \sigma \in \operatorname{Tr}_{\mathcal{A}},[\alpha+(-\alpha)](\sigma) & =\alpha(\sigma) \circ(-\alpha)(\sigma)(b y, 4) \\
& =\alpha(\sigma) \circ[\alpha(\sigma)]^{-1}(b y, 14) \\
& =i d_{\mathcal{P}} \\
& =0_{T r_{\mathcal{A}}} .(b y, 8)
\end{aligned}
$$

So exists, the additive inverse element of $\alpha$ in $E n d_{\operatorname{Tr}_{\mathcal{A}}}^{T P}$, which is $-\alpha$ for which, we have proven that it is a trace-preserving endomorphism, and we have that,

$$
\forall \alpha \in E n d_{T r_{\mathcal{A}}}^{T P}, \exists(-\alpha) \in E n d_{T r_{\mathcal{A}}}^{T P}, \alpha+(-\alpha)=0_{T r_{\mathcal{A}}} .
$$

4. $\forall \alpha, \beta \in E n d_{\operatorname{Tr}_{\mathcal{A}}}^{T P}$, have

$$
\begin{aligned}
\forall \sigma \in \operatorname{Tr}_{\mathcal{A}},[\alpha+\beta](\sigma) & =\alpha(\sigma) \circ \beta(\sigma)(b y, 4) \\
& =\beta(\sigma) \circ \alpha(\sigma)(b y, 2) \\
& =[\beta+\alpha](\sigma) .(b y, 4)
\end{aligned}
$$

So,

$$
\forall \alpha, \beta \in \operatorname{End}_{\operatorname{Tr}_{\mathcal{A}}}^{T P}, \alpha+\beta=\beta+\alpha .
$$

Theorem 5. The algebra $\left(E n d_{T r_{\mathcal{A}}}^{T P},+, \circ\right)$, is a associative unitary Ring. 
Proof. According to the Definition of a associative Unitary Ring [10], [11], [12] we are required to prove the following conditions,

(1) The grupoid $\left(E n d_{T r_{\mathcal{A}}}^{T P},+\right)$, is commutative group, we have proved this in the Theorem 7 .

(2) The action ' $\circ$ ' is associative in $E n d_{T r_{\mathcal{A}}}^{T P}$, truly, by definition of composition, we have

$$
\begin{aligned}
\forall \sigma \in \operatorname{Tr}_{\mathcal{A}},[(\alpha \circ \beta) \circ \gamma](\sigma) & =(\alpha \circ \beta)(\gamma(\sigma)) \\
& =\alpha(\beta(\gamma(\sigma))) \\
& =\alpha[(\beta \circ \gamma)(\sigma)] \\
& =[\alpha \circ(\beta \circ \gamma)](\sigma) .
\end{aligned}
$$

So we have that,

$$
\forall \alpha, \beta, \gamma \in \operatorname{End}_{\operatorname{Tr}_{\mathcal{A}}}^{T P},(\alpha \circ \beta) \circ \gamma=\alpha \circ(\beta \circ \gamma) .
$$

(3) The composition is 'distributive' according to 'addition', ie,

$$
\begin{gathered}
\forall \alpha, \beta, \gamma \in \operatorname{End}_{\operatorname{Tr}_{\mathcal{A}}}^{T P}, \\
\alpha \circ(\beta+\gamma)=\alpha \circ \beta+\alpha \circ \gamma, \text { and }(\alpha+\beta) \circ \gamma=\alpha \circ \gamma+\beta \circ \gamma
\end{gathered}
$$

Really,

$$
\begin{aligned}
\forall \sigma \in \operatorname{Tr}_{\mathcal{A}},[\alpha \circ(\beta+\gamma)](\sigma) & =\alpha[(\beta+\gamma)(\sigma)] \quad(b y, 5) \\
& =\alpha[\beta(\sigma) \circ \gamma(\sigma)](b y, 4) \\
& =\alpha[\beta(\sigma)] \circ \alpha[\gamma(\sigma)] \quad(b y, 6) \\
& =[\alpha \circ \beta](\sigma) \circ[\alpha \circ \gamma](\sigma)(b y, 5) \\
& =[\alpha \circ \beta+\alpha \circ \gamma](\sigma) . \quad(b y, 4)
\end{aligned}
$$

Hence

$$
\forall \alpha, \beta, \gamma \in \operatorname{End}_{T_{r_{\mathcal{A}}}^{T P}}, \alpha \circ(\beta+\gamma)=\alpha \circ \beta+\alpha \circ \gamma \text {. }
$$

Also,

$$
\begin{aligned}
\forall \sigma \in \operatorname{Tr}_{\mathcal{A}},[(\alpha+\beta) \circ \gamma](\sigma) & =[\alpha+\beta](\gamma(\sigma)) \quad(b y, 5) \\
& =\alpha(\gamma(\sigma)) \circ \beta(\gamma(\sigma)) \quad(b y, 4) \\
& =[\alpha \circ \gamma](\sigma) \circ[\beta \circ \gamma](\sigma)(b y, 5) \\
& =[\alpha \circ \gamma+\beta \circ \gamma](\sigma) . \quad(b y, 4)
\end{aligned}
$$

Hence

$$
\forall \alpha, \beta, \gamma \in E n d_{T_{r_{\mathcal{A}}}^{T P}}^{T P}(\alpha+\beta) \circ \gamma=\alpha \circ \gamma+\beta \circ \gamma .
$$

(4) In $E n d_{T r_{\mathcal{A}}}^{T P}$, exist the unitary element, related to composition

$$
\forall \alpha \in \operatorname{End}_{T r_{\mathcal{A}}}^{T P}, \text { have, }
$$

$$
\begin{aligned}
\forall \sigma \in \operatorname{Tr}_{\mathcal{A}},\left[\alpha \circ 1_{T r_{\mathcal{A}}}\right](\sigma) & =\alpha\left[1_{T r_{\mathcal{A}}}(\sigma)\right](b y, 5) \\
& =\alpha(\sigma)(b y, 9) .
\end{aligned}
$$


Hence

$$
\forall \alpha \in E n d_{T r_{\mathcal{A}}}^{T P}, \alpha \circ 1_{\operatorname{Tr}_{\mathcal{A}}}=\alpha,
$$

ie, the unitary element of $E n d_{T r_{\mathcal{A}}}^{T P}$, is the unitary endomorphism ' $11_{T r_{\mathcal{A}}}$ ' of $\operatorname{Tr}_{\mathcal{A}}$ on himself.

\section{References}

[1] E. Artin, Geometric algebra. New York, NY: Wiley Interscience, 1988, doi: $10.1002 / 9781118164518$

[2] M. Berger, Geometry, vol. 1. Berlin: Springer, 1987, doi: 10.1007/9783-540-93815-6

[3] M. Berger, Geometry, vol. 2. Berlin: Springer, 1987, doi: 10.1007/9783-540-93816-3

[4] H. S. M. Coxeter, Introduction to geometry, 2nd ed. New York, NY: Wiley, 1989

[5] K. Filipi, O. Zaka, and A. Jusufi, "The construction of a corp in the set of points in a line of desargues affine plane", Matematički bilten, vol. 43, no. 1, pp. 27-46, 2019, doi: 10.37560/matbil1190027k

[6] R. C. Hartshorne, Foundations of projective geometry. New York, NY: Benjamin/Cummings, 1967.

[7] D. Hilbert, The foundations of geometry, La Salle, IL: The Open Court Publishing, 1959.

[8] D. R. Hughes and F.C. Piper, Projective planes. Berlin: Spriger, 1973

[9] A. Kryftis, "Aconstructive approach to affine and projective planes", Jan. 2016. arXiv: 1601.04998v11973

[10] S. Lang, Algebra, 3rd ed. New York, NY: Springer, doi: 10.1007/978-14613-0041-0

[11] J. J. Rotman, Advanced modern algebra, 2nd ed. Providence, RI: American Mathematical Society, 2010. 
[12] R. Wisbauer, Foundations of module and ring theory. Philadelphia, PA: Gordon and Breach Science Publishers, 1991.

[13] O. Zaka, "Contribution to reports of some algebraic structures with affine plane geometry and applications", Ph. D thesis, Polytechnic University of Tirana, Department of Mathematical Engineering, 2016, doi: 10.13140/RG.2.2.13705.08804

[14] O. Zaka, "Three vertex and parallelograms in the affine plane: similarity and addition abelian groups of similarly n-vertexes in the Desargues affine plane", Mathematical modelling and applications, vol. 3, no. 1, pp. 9-15, 2018, doi: 10.11648/j.mma.20180301.12

[15] O. Zaka, "Dilations of line in itself as the automorphism of the skew-field constructed over in the same line in Desargues affine plane", Applied mathematical sciences, vol. 13, no. 5, pp. 231-237, 2019, doi: 10.12988/ams.2019.9234

[16] O. Zaka and K. Filipi, "The transform of a line of Desargues affine plane in an additive group of its points", International journal. of current research, vol. 8, no. 7, pp. 34983-34990, Jul. 2016. [On line]. Available: https://bit.ly/3hCyqpr

[17] 0. Zaka and J.F. Peters, "Isomorphic-dilations of the skew-fields constructed over parallel lines in the desargues affine plane", Mar. 2019, arXiv: $1904.01469 \mathrm{v} 1$

[18] O. Zaka and J.F. Peters, "Ordered line and skew-fields in the Desargues affine plane", May 2019, arXiv:1905.03859

[19] O. Zaka, "One construction of an affine plane over a corps", Journal of advances in mathematics, vol. 12, no. 5, pp. 6200-6206, 2016, doi: 10.24297/jam.v12i5.215

[20] O. Zaka, "A description of collineations-groups of an affine plane", Libertas mathematica, vol. 37, no. 2, pp. 81-96, 2017. [On line]. Available: https://bit.ly/3ftAY7w 